\title{
A novel design of 5-input majority gate in quantum-dot cellular automata technology
}

\begin{abstract}
Quantum-dot Cellular Automata (QCA) technology is one of the most important technologies, which can be suitable replacement for conventional technologies at Nano-scale. The principle logic elements in the QCA technology are majority gates and inverters. In this paper, a novel design is proposed for 5-input majority gate in the QCA technology. The proposed 5-input majority gate uses half distance. The QCADesigner tool version 2.0.3 is utilized for verifying functionality and layout of the proposed majority gate. The simulation results demonstrate that the proposed 5-input majority gate design provides significant improvements in the logical circuit design in terms of area and the number of required cells in comparison with other majority gates.
\end{abstract}

Keyword: 5-input majority gate; Nanotechnology; QCADesigner; Quantum-dot cellular automata 